\title{
Histology of variants of urothelial carcinoma of the bladder: a Moroccan series of 39 cases
}

\author{
Amine Saouli, ${ }^{*}$ Tarik Karmouni, Khalid El Khader, Abdellatif Koutani and Ahmed Iben Attya Andaloussi
}

Background: The variant of urothelial carcinoma (UCV) is a rare and aggressive histological entity; its therapeutic management remains debated.

Methods: Between January 2017 and October 2019, 39 patients were diagnosed retrospectively with urothelial carcinoma with a histological variant. The aim of this study was to analyze the clinico-pathological and therapeutic aspects of these variants to better understand this invasive disease.

Results: In 39 cases, 36 (92\%) were men and 3 (8\%) were women; the average age was 62 years; $54 \%$ of patients had $a \geq$ CT3 stage with clinical lymph node involvement in $28 \%$ and $23 \%$ had distant metastases, $36 \%$ of patients were treated with RC and $54 \%$ by other therapeutic and $10 \%$ refused RC. No patient received neoadjuvant chemotherapy. Lymph node involvement was $28.5 \%$. The two predominant histological types were squamous and micropapillary differentiation between the operating piece and bladder biopsy $28.5 \%, 30 \%$ and $21.5 \%$ and $13 \%$, respectively. The overall survival at 20 months was 70 and $40 \%$ for RC and the other therapeutic (TURB alone or a TURB with an adjuvant), respectively. There was no significant difference in survival between the two groups ( $p=0.27$ ).

Conclusion: UCVs are heterogeneous groups of tumors which are increasingly identified due to their distinct morphological characteristics with variable clinical prognosis. Therapeutic management is not in favor of cystectomy in our study. However, a prospective study using a larger cohort is still necessary to validate our results.

Keywords: Urothelial carcinoma, Variant, Overall survival, Radical cystectomy

\section{Background}

Urothelial bladder carcinoma (UBC) is well known for its many histological variants [1]. About $80 \%$ of the UBC is a pure or conventional urothelial carcinoma (UC), while the remaining $20 \%$ is represented by a divergent histological differentiation. The pathophysiological study of these variants has recently aroused growing interest, because the therapeutic approach is not uniform for all entities, for example, the effectiveness of NAC in the neuroendocrine variant [2-5].

These variants have important diagnostic, prognostic and therapeutic implications.

The diagnosis of these entities requires expertise to better identify them. In one study, 589 samples of

\footnotetext{
*Correspondence: amine.saouli0@gmail.com Department of Urology B, CHU Ibn Sina, Faculty of Medicine and Pharmacy, Mohamed V University, Rabat, Morocco
}

transurethral resection of the bladder (TURB) interpreted by expert genitourinary pathologists confirmed the presence of a histological variant which was not reported by general pathologists in $44 \%$ of the cases [6].

In the future, a molecular and genetic study of these variants will allow them to be better defined.

An accurate diagnosis allows you to stratify the risks, determine the prognosis and choose an appropriate treatment. The published data come from small retrospective series and extrapolation of data from pure UC; this is due to the rarity of certain subgroups and the exclusion of patients with a histological variant from clinical studies [7].

The treatment of impure UC remains controversial; there is no therapeutic consensus in current practice.

These entities are frequently associated with a poor prognosis. Some authors suggest that impure UC is more aggressive than pure or conventional UC [8], although 
many authors show that the prognosis after radical cystectomy is identical in the two groups [9].

The aim of this study is to analyze the clinico-pathological and therapeutic aspects of these variants to better understand this invasive disease.

\section{Methods}

Between January 2017 and October 2019, a retrospective monocentric study including 39 patients with urothelial carcinoma with a histological variant.

Our patients were treated by a radical cystectomy (RC) or a transurethral resection of the bladder (TURB) alone or a TURB with an adjuvant treatment (systemic chemotherapy or endo-vesical immunotherapy type BCG therapy).

\subsection{Inclusion criteria}

This study included all patients admitted to the urology department for the treatment of bladder cancer with a histological variant, whether endoscopically or by open surgery.

All histological differentiations were included.

\subsection{Exclusion criteria}

The following were excluded from our study:

- Patients receiving endoscopic treatment for another pathology

- Patients refusing the study

- Patients with pure urothelial carcinoma.

\subsection{Statistical analysis}

A rigorous statistical methodology has enabled the collection of data and parameters to study various clinical records thanks to an operating sheet including radiological imaging and standardized histological reports according to the guidelines of the French pathology association.

Kaplan-Meier curves were used to assess the impact of different treatments on overall survival. The log-rank test was used to compare these different curves.

The capture of the results, the evaluation of the various statistical indices were carried out using the software SPSS version 10 . The results are considered statistically significant for a $p<0.05$.

Consent was systematic for all patients included in the study.

\section{Results}

The details of the clinical and paraclinical parameters of our patients will be set out retrospectively and successively in the tables and figure below.
We collected 39 patients treated for bladder cancer with a histological variant.

\subsection{The clinical characteristics of the patients}

The mean age was 62 years, with a male predominance $92 \%$ versus $8 \%$ of the female.

Hydronephrosis was observed in $33.5 \%$ of our patients, 30 (77) patients were smoking and 13 (33.5) patients had an ECOG PS $\geq 2$.

Fifty-four percentage of patients had $\mathrm{a} \geq \mathrm{cT} 3$ stage with clinical lymph node involvement of $28 \%$ and at the initial diagnosis, $23 \%$ of patients had distant metastases (Table 1).

No patient received neoadjuvant chemotherapy.

\subsection{The therapeutic characteristics of the patients}

The surgical procedure corresponded in $36 \%$ of the patients to a RC always associated with an extensive pelvic lymph node dissection, including the obturator, external iliac, internal iliac and primitive distal iliac regions going up to the crossing of the ureters.

TURB alone was performed in 3\% of our patients, $28 \%$ had TURB with systemic chemotherapy such as MVAC (methotrexate, vinblastine, doxorubicin and cisplatin), $23 \%$ had TURB with BCG therapy and 10\% refused treatment radical (Table 1 ).

No patient was treated with radiotherapy using a multimodal approach.

\subsection{The pathological and oncological results of the patients}

The ISC was present in the bladder piece in $21.5 \%$ against $38 \%$ in the bladder biopsy, lymphovascular invasion (LVI) was present in the bladder piece in $28.5 \%$ compared to $46 \%$ in the bladder biopsy, and lymph node invasion was $28.5 \%$.

The two predominant histological variants were squamous and micropapillary differentiation between the bladder piece and bladder biopsy $28.5 \%, 30 \%$ and $21.5 \%$ and $13 \%$, respectively. The percentage of the micropapillary component $\geq 15 \%$ was present in $5 \%$ of the patients in the bladder biopsy against $7 \%$ in the bladder piece (Table 2).

Mixed differentiations were present in the bladder piece in $21 \%$ compared to $15 \%$ in the bladder biopsy.

The overall survival at 20 months was $70 \%$ and $40 \%$ for the patients who had RC and the other therapeutic (TURB alone or a TURB with an adjuvant), respectively; there was no significant difference in survival between the two groups ( $p=0.27$ ) (Fig. 1). 
Table 1 The clinical characteristics of the patients in our series

\begin{tabular}{|c|c|}
\hline & $\begin{array}{l}\text { Study } \\
\text { population } \\
(n=39)\end{array}$ \\
\hline \multicolumn{2}{|l|}{ Sex, $n(\%)$} \\
\hline Male & $36(92)$ \\
\hline Female & $3(8)$ \\
\hline Mean age, (years) & 62 \\
\hline \multicolumn{2}{|l|}{ Tobacco, $n(\%)$} \\
\hline Yes & $30(77)$ \\
\hline No & $9(23)$ \\
\hline \multicolumn{2}{|l|}{ ECOG PS, $n(\%)$} \\
\hline 0 & $9(23)$ \\
\hline 1 & $17(43.5)$ \\
\hline$\geq 2$ & $13(33.5)$ \\
\hline \multicolumn{2}{|l|}{ Hydronephrosis, n (\%) } \\
\hline Present & $17(33.5)$ \\
\hline Absent & $22(56.5)$ \\
\hline \multicolumn{2}{|l|}{ Clinical stage T, $n$ (\%) } \\
\hline $\mathrm{T} 1$ & $2(5)$ \\
\hline $\mathrm{T} 2$ & $2(5)$ \\
\hline T3 & $16(41)$ \\
\hline T4 & $9(23)$ \\
\hline Tx & $10(26)$ \\
\hline \multicolumn{2}{|l|}{ Lymph node clinical stage $\mathrm{N}, n(\%)$} \\
\hline No & $18(46)$ \\
\hline N1 & $6(15)$ \\
\hline $\mathrm{N} 2$ & $5(13)$ \\
\hline $\mathrm{Nx}$ & $10(26)$ \\
\hline \multicolumn{2}{|l|}{ Visceral metastases, $n$ (\%) } \\
\hline Yes & $9(23)$ \\
\hline No & $30(77)$ \\
\hline \multicolumn{2}{|l|}{ Treatment received, $n$ (\%) } \\
\hline TURB alone & $1(3)$ \\
\hline TURB + systemic chemotherapy & $11(28)$ \\
\hline TURB + BCG therapy & $9(23)$ \\
\hline $\mathrm{RCP}$ & $14(36)$ \\
\hline Refusal of radical treatment & $4(10)$ \\
\hline Mean follow-up, (months) & 18 \\
\hline
\end{tabular}

ECOG PS Eastern Cooperative Oncology Group performance status, TURB transurethral resection of the bladder, $R C P$ radical cystoprostatectomy

\section{Discussion}

Variants of UC tend to be underdiagnosed or misclassified for several reasons [3].

Tumors are heterogeneous and subsampling can compromise the histological recognition of these entities. This explains the variable agreement rate between the specimen of TURB and radical cystectomy (RC) reported in the literature; while some studies show relatively low agreement $[4,5]$, others report such high rates $83.6 \%$ [10]. In our study was able to show a $36 \%$ agreement rate.

However, the histology of these variants is difficult to interpret due to the heterogeneity and the percentage of each variant. In addition, it is not uncommon to have several types of variants in the same tumor.

The therapeutic management of various histological variants remains controversial [11]. It is essentially based on the identification of subtypes responding to non-surgical treatment (systemic therapy, intravesical therapy, radiotherapy, etc.) before radical treatment [12].

The role of neoadjuvant chemotherapy (NAC) in the therapeutic arsenal of these variants has shown an improvement in overall survival mainly in the neuroendocrine component and a decrease in the tumor stage compared to other differentiations.

Vetterlein et al. reported significant overall survival in patients with neuroendocrine bladder tumors who received NAC. In addition, patients with micropapillary differentiation and sarcomatoid differentiation have shown a decrease in the disease stage pT3 and/or pN1 when they received NAC [13]. Neoadjuvant chemotherapy has not been used in our population.

The micropapillary component of these variants does not confer a worse prognosis than pure UC in terms of overall survival after total cystectomy. However, NAC was not significantly associated with better survival outcomes, and it resulted in a significant rate of decline in the stage of the disease [14].

Although there is no clear recommendation in the management of these entities, several studies have proven the place of total cystectomy.

Veskimäe et al. reported that in the majority of these variants, such as the micropapillary and plasmocytoid component, the treatment is mainly $\mathrm{NAC}$ and radical cystectomy [15].

A meta-analysis published in 2017 reported that the $\mathrm{UC}$ variants did not predict a poor prognosis for patients treated with RC. However, the data have shown patients with these variants who receive $\mathrm{RC}$ as soon as possible for better results [16].

Stroman et al. reported a decrease in overall survival in patients with histological variants at $14 \%$ at 2 years and $23 \%$ at 5 years after RC compared to pure UC. Patients should be informed about the high risk and unpredictable nature of these entities and the recommendation for the first RC performed for this indication [17].

The overall survival at 20 months in our study was $70 \%$ and $40 \%$ for the patients who had RC and the other therapeutic (TURB alone or a TURB with an adjuvant), respectively.

Regarding BCG immunotherapy, Gofrit et al. reported that patients treated with BCG intravesical 
Table 2 The pathological characteristics of the bladder biopsy and the operating room of the patients in our series

\begin{tabular}{|c|c|c|}
\hline & $\begin{array}{l}\text { Bladder biopsy } \\
N=39\end{array}$ & $\begin{array}{l}\mathrm{RC} \\
N=14\end{array}$ \\
\hline \multicolumn{3}{|l|}{ Pathological stage pT, $n(\%)$} \\
\hline $\mathrm{Ta}$ & $4(10)$ & $0(0)$ \\
\hline $\mathrm{T} 1$ & $18(46)$ & $0(0)$ \\
\hline $\mathrm{T} 2$ & $17(44)$ & $6(43)$ \\
\hline T3 & - & $6(43)$ \\
\hline $\mathrm{T} 4$ & - & $2(14)$ \\
\hline Pathological lymph node stage pN, $n$ (\%) & - & \\
\hline No & & $6(43)$ \\
\hline N1 & & $3(21.5)$ \\
\hline N2 & & $2(14)$ \\
\hline N3 & & $0(0)$ \\
\hline $\mathrm{Nx}$ & & $3(21.5)$ \\
\hline \multicolumn{3}{|l|}{ ISC, $n(\%)$} \\
\hline Present & $15(38)$ & $3(21.5)$ \\
\hline Absent & $24(62)$ & $11(78.5)$ \\
\hline \multicolumn{3}{|l|}{ Lymphovascular invasion, $n$ (\%) } \\
\hline Present & $18(46)$ & $4(28.5)$ \\
\hline Absent & $21(54)$ & $10(71.5)$ \\
\hline \multicolumn{3}{|l|}{ Histological type, $n(\%)$} \\
\hline Malpighian differentiation & $12(30)$ & $4(28.5)$ \\
\hline Micropapillary differentiation & $5(13)$ & $3(21.5)$ \\
\hline Glandular differentiation & $6(15)$ & $0(0)$ \\
\hline Microcystic differentiation & $1(2.5)$ & $0(0)$ \\
\hline Sarcomatoid differentiation & $2(5)$ & $2(14)$ \\
\hline Lympho-epithelial differentiation like & $2(5)$ & $1(7)$ \\
\hline Neuroendocrine differentiation & $1(2.5)$ & $1(7)$ \\
\hline Plasmocytoid differentiation & $2(5)$ & $0(0)$ \\
\hline Like lympho-epithelial differentiation + sarcomatoid + microcystic & $1(2.5)$ & $1(7)$ \\
\hline Glandular + like lympho-epithelial differentiation & $1(2.5)$ & $0(0)$ \\
\hline Malpighian + glandular differentiation & $2(5)$ & $0(0)$ \\
\hline Squamous + micropapillary differentiation & $1(2.5)$ & $1(7)$ \\
\hline Sarcomatoid + glandular differentiation + microcystic & $1(2.5)$ & $1(7)$ \\
\hline No differentiation & $2(5)$ & $0(0)$ \\
\hline Lymph node invasion pN, $n$ (\%) & - & $5(35.5)$ \\
\hline \multicolumn{3}{|l|}{ Micropapillary component, (\%) } \\
\hline 5 & $2(5)$ & $1(7)$ \\
\hline 10 & $1(2.5)$ & $1(7)$ \\
\hline$\geq 15$ & $2(5)$ & $1(7)$ \\
\hline
\end{tabular}

$R C P$ radical cystoprostatectomy, ISC in situ carcinoma

immunotherapy did not show efficacy even when confirmation of diagnosis with re-evaluation TURB and rigorous follow-up. The progression rate of these patients is high ( $40 \%$ at 5 years compared to $17.5 \%$ pure high grade UC). Patients treated with BCG intravesical immunotherapy have a $27 \%$ chance of dying from this disease within 5 years, compared with $7.5 \%$ chance of high grade UC. As such, these patients should be informed of this adverse clinical course and considerations for cystectomy are strongly recommended [9].

A recent study of 114 phase 2 patients (PURE-01) administered to patients with impure UC three cycles of $200 \mathrm{mg}$ of pembrolizumab preceding a RC. The preliminary results of this study confirm the activity of pembrolizumab in neoadjuvant the treatment of $\mathrm{UC}$ infiltrating the muscle with a histological variant [18]. 


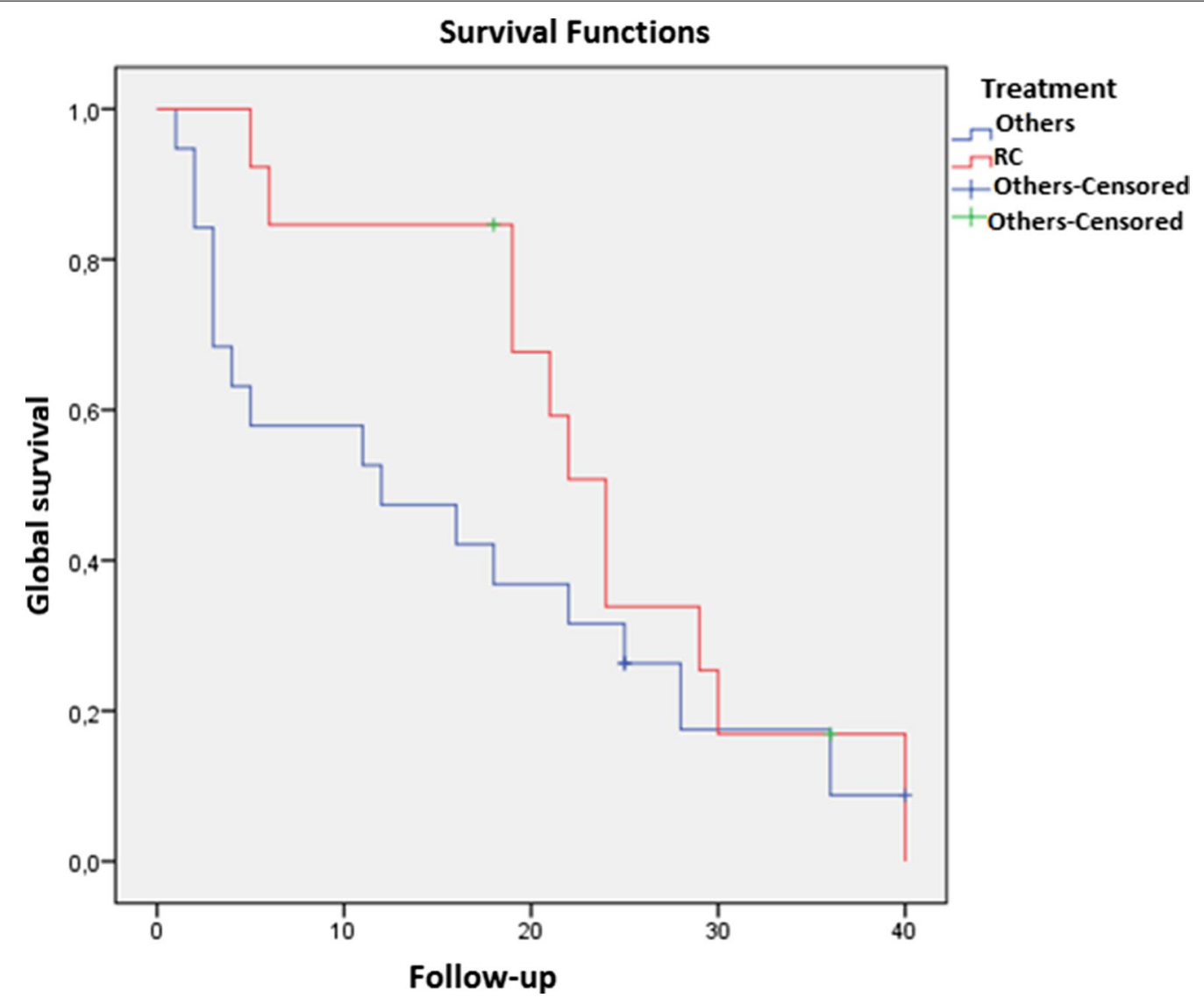

Fig. 1 Represents the overall survival rate in the two groups as a function of time (months) according to Kaplan-Meier

The limitations of this study were mainly the reduced number of patients and the selection bias by default of randomization. Finally, the heterogeneity of both histological variants of urothelial carcinoma of the bladder and also the use of curative therapies are important limits to emphasize. These results are well worth investigating in multicentric prospective studies with a larger sample.

\section{Conclusion}

UCVs are heterogeneous groups of tumors which are increasingly identified due to their distinct morphological characteristics with variable clinical prognosis.

Therapeutic management is not in favor of cystectomy in our study. However, a prospective study using a larger cohort is still necessary to validate our results.

Future studies are needed to assess the reactivity of different histological variants to new immunotherapy agents.

\section{Abbreviations}

UBC: urothelial bladder carcinoma; UC: urothelial carcinoma; TURB: transurethral resection of bladder; $R C$ : radical cystectomy; $B C G$ : Bacilli Calmette and
Guerin; ECOG PS: The Eastern Cooperative Oncology Group performance status; ISC: in situ carcinoma; LVI: lymphovascular invasion; NAC: neoadjuvant chemotherapy.

\section{Acknowledgements \\ Not applicable.}

Authors' contributions

A.S wrote the article, K.T, K.E, A.K and A.I corrected the article. All authors read and approved the final manuscript.

\section{Funding}

No funding.

\section{Ethics approval and consent to participate}

This study was approved by the ethics committee of Ibn Sina Hospital in Mohamed V University. The patients provided verbal consent to participate in this study.

\section{Consent for publication}

The patient gave his informed and free consent for the publication of this study.

\section{Competing interests}

The authors declare that they have no conflicts of interest in connection with this article.

Received: 24 July 2020 Accepted: 14 December 2020

Published online: 07 January 2021 


\section{References}

1. Moch H, Cubilla AL, Humphrey PA et al (2016) The 2016 who classification of tumours of the urinary system and male genital organs-part A: renal, penile, and testicular tumours. Eur Urol 70:93-105

2. Kaimakliotis HZ, Monn MF, Cary KC et al (2014) Plasmacytoid variant urothelial bladder cancer: is it time to update the treatment paradigm? Urol Oncol 32:833-838

3. Porten SP, Willis D, Kamat AM (2014) Variant histology: role in management and prognosis of nonmuscle invasive bladder cancer. Curr Opin Urol 24:517-523

4. Xylinas E, Rink M, Robinson BD et al (2013) Impact of histological variants on oncological outcomes of patients with urothelial carcinoma of the bladder treated with radical cystectomy. Eur J Cancer 49:1889-1897

5. Monn MF, Kaimakliotis HZ, Pedrosa JA et al (2015) Contemporary bladder cancer: variant histology may be a significant driver of disease. Urol Oncol 33(18):e15-2

6. Shah RB, Montgomery JS, Montie JE, Kunju LP (2013) Variant (divergent) histologic differentiation in urothelial carcinoma is under-recognized in community practice: impact of mandatory central pathology review at a large referral hospital. Urol Oncol 31:1650-1655

7. Lobo N, Shariat SF, Guo CC, Fernandez MI, Kassouf W, Choudhury A, Gao J, Williams SB, Galsky MD, Taylor JA 3rd, Roupret M, Kamat AM (2020) What is the significance of variant histology in urothelial carcinoma? Eur Urol Focus 6(4):653-663

8. Black PC, Brown GA, Dinney CP (2009) The impact of variant histology on the outcome of bladder cancer treated with curative intent. Urol Oncol 27(1):3-7

9. Gofrit Ofer N, Yutkin Vladimir, Shapiro Amos, Pizov Galina, Zorn Kevin C, Hidas Guy, Gielchinsky Ilan, Duvdevani Mordechai, Landau Ezekiel H, Pode Dov (2016) The response of variant histology bladder cancer to intravesical immunotherapy compared to conventional cancer. Front Oncol 15(6):43

10. Sahin AA, Myhre M, Ro JY et al (1991) Plasmacytoid transitional cell carcinoma. Report of a case with initial presentation mimicking multiple myeloma. Acta Cytol 35:277-280

11. Keck B, Wach S, Stoehr R et al (2013) Plasmacytoid variant of bladder cancer defines patients with poor prognosis if treated with cystectomy and adjuvant cisplatin-based chemotherapy. BMC Cancer 13:71

12. Li Q, Assel M, Benfante NE, Pietzak EJ, Herr HW, Donat M, Cha EK, Donahue TF, Bochner BH, Dalbagni G (2019) The impact of plasmacytoid variant histology on the survival of patients with urothelial carcinoma of bladder after radical cystectomy. Eur Urol Focus 5(1):104-108

13. Vetterlein MW, Wankowicz SAM, Seisen T, Lander R, Löppenberg B, Chun FK, Menon M, Sun M, Barletta JA, Choueiri TK, Bellmunt J, Trinh QD, Preston MA (2017) Neoadjuvant chemotherapy prior to radical cystectomy for muscle-invasive bladder cancer with variant histology. Cancer 123(22):4346-4355

14. Abufaraj M, Foerster B, Schernhammer E, Moschini M, Kimura S, Hassler MR, Preston MA, Karakiewicz PI, Remzi M, Shariat SF (2019) Micropapillary urothelial carcinoma of the bladder: a systematic review and metaanalysis of disease characteristics and treatment outcomes. Eur Urol 75(4):649-658

15. Veskimäe E, Espinos EL, Bruins HM, Yuan Y, Sylvester R, Kamat AM, Shariat SF, Witjes JA, Compérat EM (2019) What Is the prognostic and clinical importance of urothelial and nonurothelial histological variants of bladder cancer in predicting oncological outcomes in patients with muscle-invasive and metastatic bladder cancer? A European Association of Urology Muscle Invasive and Metastatic Bladder Cancer Guidelines Panel systematic review. Eur Urol Oncol 2(6):625-642

16. Chen Q, Li L, Wang G, Hu J, Sun T, Fu B (2017) Do histological variants in urothelial carcinoma of the bladder portend poor prognosis? A systematic review and meta-analysis. Oncotarget 8:48263-48271

17. Stroman L, Nair R, Russell B, Malik N, Desai A, Chandra A, Thurairaja R, Dasgupta P, Khan MS, Malde S (2019) The impact of non-urothelial variant histology on oncological outcomes following radical cystectomy. BJU Int 124(3):418-423

18. Necchi A, Raggi D, Gallina A, Madison R, Colecchia M, Lucianò R, Montironi R, Giannatempo P, Farè E, Pederzoli F, Bandini M, Bianchi M, Colombo R, Gandaglia G, Fossati N, Marandino L, Capitanio U, Dehò F, Ali SM, Chung JH, Ross JS, Salonia A, Briganti A, Montorsi F (2020) Updated results of PURE-01 with preliminary activity of neoadjuvant pembrolizumab in patients with muscle-invasive bladder carcinoma with variant histologies. Eur Urol 77(4):439-446

\section{Publisher's Note}

Springer Nature remains neutral with regard to jurisdictional claims in published maps and institutional affiliations.

\section{Submit your manuscript to a SpringerOpen ${ }^{\circ}$ journal and benefit from:}

- Convenient online submission

- Rigorous peer review

- Open access: articles freely available online

- High visibility within the field

- Retaining the copyright to your article

Submit your next manuscript at springeropen.com 